\title{
Glutathione S-transferase Al suppresses tumor progression and indicates better prognosis of human primary hepatocellular carcinoma
}

Xiaojia Liu ${ }^{1 \#, ~ X i a n x i a n ~ S u i " \#, ~ C a n j i n g ~ Z h a n g ~}{ }^{3}$, Kelu Wei $^{4}$, Yun Bao ${ }^{1}$, Ji Xiong ${ }^{1}$, Zhongwen Zhou ${ }^{1}$, Zhongqing Chen ${ }^{1}$, Chaoqun Wang ${ }^{5}$, Hongguang Zhu ${ }^{1,6}$, Feng Tang ${ }^{1 凶}$

1. Division of Surgical Pathology, Huashan Hospital, Fudan University, Shanghai 200040, China

2. Laboratory of Medical Molecular Biology, Experimental Teaching Center, School of Basic Medical Sciences, Fudan University, Shanghai 200032, China

3. Key Laboratory of Medical Molecular Virology, the Institutes of Biomedical Sciences, Fudan University, Shanghai 200032, China

4. Shanghai Medical College, Fudan University, Shanghai 200032, China

5. Department of General Surgery, Huashan Hospital, Fudan University, Shanghai, 200040, China

6. Department of Pathology, School of Basic Medical Sciences, Fudan University, Shanghai 200032, China

\#These authors contributed equally to this work.

$\triangle$ Corresponding author: Feng Tang MD., Ph.D., Division of Surgical Pathology, Huashan Hospital, Fudan University, 12 Urumqi Middle Road, 200040, Shanghai, China. E-mail: tangfeng1966@sohu.com.

(C) The author(s). This is an open access article distributed under the terms of the Creative Commons Attribution License (https://creativecommons.org/licenses/by/4.0/). See http://ivyspring.com/terms for full terms and conditions.

Received: 2019.05.08; Accepted: 2019.09.19; Published: 2020.01.01

\begin{abstract}
Glutathione S-transferase (GST) family members play an important role in detoxification, metabolism and carcinogenesis. The aim of this study is to investigate the effect of Glutathione S-transferase $\mathrm{Al}$ (GSTAl) on the prognosis of $\mathrm{HCC}$ and to understand its role in tumor progression and the possible mechanism. GSTAl in HCC was assessed using immunohistochemical staining, and it was found that HCC patients with better pathological differentiation had higher GSTAI abundance. Further, high GSTAl expression was correlated with low AFP, absent PVTT, and early stage TNM for HCC patients. Higher GSTAl indicated longer overall survival and disease-free survival, while lower GSTAl indicated poorer prognosis. Subsequently, lentiviral vector carrying GSTAl gene was successfully constructed and maintained high expression in 97H and SNU449 liver cancer cells. We found that high GSTAI restrained liver cancer cell proliferation, migration and invasion in vitro. Western blot showed that LKBI and p-AMPK were upregulated while p-mTOR, P-P70 S6 Kinase and MMP-9 were downregulated in high GSTAl groups. Taken together, high GSTAl correlated with satisfactory prognosis of HCC. Additionally, GSTAl may act as a protective factor through suppression of tumorigenesis by targeting AMPK/mTOR in HCC.
\end{abstract}

Key words: hepatocellular carcinomas; glutathione S-transferase A1 protein; prognosis; cellular proliferation; metastasis; AMP-Activated Protein Kinases

\section{Introduction}

Primary hepatic cancer is the third leading cause of cancer-related mortality in China ${ }^{1}$. The most frequently occurring hepatic cancer is hepatocellular carcinoma (HCC), which accounts for $90-95 \%$ of all primary liver cancers and causes more than 234,000 deaths each year. Glutathione S-transferases (GSTs) are isoenzymes that have overlapping substrate specificities and protect cells from cytotoxic and carcinogenic agents ${ }^{2}$. Eight isoforms of cytosolic-soluble GSTs have been recognized in humans, including $\alpha, \kappa, \mu, \Pi, \sigma, \theta, \zeta$, and $\omega^{3,4}$. Glutathione $S$-transferase a1 (GSTA1, Gene ID: 2938) has shown both stimulatory5-8 and inhibitory effects $^{9-12}$ on tumorigenesis. The association between genetic polymorphism of GSTA1 and susceptibility to cancer has been discussed in previous studies ${ }^{13-15}$, but 
unfortunately, the underlying mechanism has remained unclear. In this study, we aimed to clarify GSTA1's effect on HCC prognosis and to determine its role in tumor progression.

There were two phases of our GSTA1 investigation. In the first phase, we employed immunohistochemistry (IHC) to determine GSTA1 protein abundances in HCC tissues, and analyzed their correlations to HCC clinicopathological characteristics. We also studied the prognostic impact of GSTA1 with Kaplan-Meier survival curves and Cox regression analyses on HCC patients. In the second phase, we performed functional analysis by altering GSTA1 expression in liver cancer cells and performed cytology experiments to characterize its biological role in HCC progression and investigated the underlying mechanism.

\section{Materials and Methods}

\section{Ethics statement}

This investigation was conducted in accordance with the Declaration of Helsinki and guidelines approved by the Institutional Review Board of Huashan Hospital, with ratification from the National Natural Science Foundation of China (NSF No. 81902834).

\section{Patient selection}

A total of 90 HCC patients who underwent hepatectomies during the year of 2011 were randomly selected from Huashan Hospital in our study. The criteria for case selection were as follows: (1) pathological diagnosis of $\mathrm{HCC}$, (2) no anti-cancer therapies received prior to surgery, and (3) no history of other cancer. Tumor stage was defined according to the American Joint Committee on Cancer (AJCC, 2018-01-01， 8th edition) Tumor-Node-Metastasis (TNM) staging system. Tumor grade was assigned by the Edmondson-Steiner grading system.

All the patients were being regularly followed for up to 72 months, with a median survival time of 51 months (range, 1-72 months). The overall survival (OS) was defined as the length of time between the surgery and death, or the last follow-up examination. Disease-free survival (DFS) was calculated from the date of tumor resection until detection of tumor recurrence.

\section{TMA and Immunohistochemistry}

Tissue microarray (TMA) was constructed and the slides were incubated with the rabbit polyclonal primary antibody against GSTA1 (NBP-33586, 1:2000 dilution, Novusbio, USA) overnight at $4^{\circ} \mathrm{C}$ in a moist chamber, and then conjugated with secondary antibody (NB7156, 1:2000 dilution; Novusbio, USA) for 60 minutes at room temperature. Finally, the slides were stained for 15 seconds using the DAB Kit (Boster Bio-Engineering Company, Wuhan, China). DAB staining regions for GSTA1 were scored by two pathologists blinded to the clinical parameters.

The score standard for the staining intensity was as follows: 0(negative), 1 (weak), 2 (moderate), 3 (strong). The score of staining extent was $1(<25 \%), 2$ $(25 \%-50 \%), 3(51 \%-75 \%)$, and $4(>75 \%)$. The final GSTA1 expression score was calculated with the intensity score $\times$ extent score, ranging from 0 to $12^{16}$. The staining results were divided into 3 categories based on the sum of scores: 0-3 was low GSTA1 group, 4-8 was moderate GSTA1 group, 9-12 was high GSTA1 group.

\section{Cell culture and transfection}

Human liver cancer cell lines (HepG2, 97H, SMMC-7721, HCC-LM3, PLC-PRF5, SK-Hep1 and SNU449) were obtained from Shanghai Institute of Cell Biology, Chinese Academy of Sciences. SNU449 was cultured in RPMI-1640 (Lot.1869036, Gibco, US), while others were cultured in Dulbecco's Modified Eagle Medium (DMEM, Cat No. 8113262, Gibco, US), supplemented with $10 \%$ fetal bovine serum (FBS, Lot.1438121, Gibco, US), penicillin $(100 \mathrm{U} / \mathrm{ml}$, Cat No.15140-122, Invitrogen, US), and streptomycin (100 $\mathrm{mg} / \mathrm{ml}$, Cat No.15140-122, Invitrogen, US), at $37^{\circ} \mathrm{C}$ in a $5 \%$ humidified $\mathrm{CO}_{2}$ incubator. The lentivirus vectors for upregulation of GSTA1 were obtained from GenePharma Co., Ltd (Shanghai, China). Liver cancer cells were infected with the lentivirus vectors constitutively expressing GSTA1 or empty vectors, according to the procedures of the manufacturer. Transfection efficacy was confirmed by western blot.

\section{Cell proliferation assay}

The effect of GSTA1 on liver cancer cell proliferation was detected by CCK8. Cells in the logarithmic phase of growth were seeded in 96-well plates $\left(1 \times 10^{3} /\right.$ well) and cultured for $24,48,72$ and 96 hours. Subsequently, $10 \mu \mathrm{L}$ of CCK-8 solution (Dojindo Laboratories, Kumamoto, Japan) were added into each well and incubated for 2 hours. Optical density (OD) was measured at a wavelength of $450 \mathrm{~nm}$ by an automatic microplate reader (Bio Tek, USA). As for colony formation assay, cells were placed in six-well plates at $2 \times 10^{3} /$ well in triplicate and were routinely cultured for 2 weeks. Colonies were fixed by paraformaldehyde, stained in crystal violet, then photographed. The colonies were lysed with glacial acetic acid solution (GAAS, Cat No. 537020, Sigma-Aldrich, US), and the lysate concentration was quantified at an absorbance of OD560 nm using an automatic microplate reader ${ }^{17}$. 


\section{Migration and Invasion assay}

Transwell chambers were used to evaluate migration and invasion ability. For the migration assay, $5 \times 10^{4}$ tumor cells were seeded in the upper chamber with serum free medium, while the lower chamber contained 10\% FBS medium, and incubated for $24 \mathrm{~h}$. For the invasion assay, the inserts were pre-coated with extracellular matrigel $(2 \mu \mathrm{g} / \mu \mathrm{L}, \mathrm{BD}$ USA) at $37^{\circ} \mathrm{C}$ for one hour and then $5 \times 10^{4}$ cells were seeded into each well. 24 hours later, the media was discarded and the upper chamber was washed with PBS three times. The membrane was fixed in $4 \%$ polycondensation formaldehyde solution for $10 \mathrm{~min}$ and then stained with $0.5 \%$ crystal violet for $20 \mathrm{~min}$, washed with water and dried $24 \mathrm{~h}$ before imaging. A 400-fold inversion microscope was used to count cells that moved to the sublayer of polycarbonate membranes.

\section{Western Blot Assay}

Protein samples were extracted and then separated with SDS-PAGE and transferred onto nitrocellulose membranes (Bio-Rad, Hercules, USA). The membranes were blocked with 5\% non-fat milk in Tris-buffered saline (TBS) containing $0.1 \%$ Tween-20 for 2 hours at room temperature. The blots were probed with the relevant primary antibodies overnight at $4^{\circ} \mathrm{C}$, and then probed with a secondary antibody for 1 hour. An enhanced chemiluminescence detection method (Pierce ECL Western Blotting Substrate, Thermol, USA) was used to visualize the blots. Anti-GSTA1 (NBP-33586, 1:1000 dilution) was purchased from Novusbio company, USA. Other primary antibodies were purchased from CST company, including anti-AMPK a (\#2795), anti-p-AMPK a Thr172 (\#50081), anti-p-mTOR Ser2448 (\#2971), anti-p-p70 S6 Kinase (\#9204), anti-MMP-9 (\#13667), and anti-LKB1 (\#3050). Anti- $\beta$-actin (\#3700) was used as the internal control antibody.

\section{Statistical Analysis}

All experiments were repeated at least three times. Data were analyzed with SPSS software and expressed as mean $\pm \mathrm{SD}$. $P<0.05$ was considered statistically significant. GSTA1 abundances between tumor and para-tumor tissue were analyzed by Wilcoxon signed-rank test. Kruskal-Wallis one-way analysis of variance (ANOVA) was performed to determine the relevance between GSTA1 and clinicopathological variables of HCC patients. Kaplan-Meier and log-rank test analyses were performed to determine the effect of GSTA1 on HCC patient survival. Multivariate Cox proportional hazard regression model was used to assess the prognostic variables in HCC. Mann-Whitney U test was performed to compare the variables of two groups in CCK8 assay, colony formation assay, invasion and migration assay, and western blot.

\section{Results}

\section{High GSTAl correlated with well-differentiation and early stage of HCC}

IHC results indicated that GSTA1 was high in para-tumor tissues compared with that in HCC tissues $(P<0.05$, Figure $1 \mathrm{~A})$. We also found that GSTA1 was related to the differentiation degree of HCC. The better the differentiation, the higher the expression of GSTA1, and vice versa (Figure 1B). And it was also very interesting that liver cancer cells with lower malignancy and weaker metastasis ability (including HepG2 and PLC-PRF5) had higher GSTA1 compared with other cells, which showed higher malignancy and strong metastasis ability (Figure 1C).

Further, our clinicopathologic characteristics study showed that high GSTA1 was correlated with low serum AFP, absence of PVTT, and early stage of TNM (Table 1 , all $P<0.05$ ). However, GSTA1 was not related to HCC patients' age, gender, $\mathrm{HBsAg}$, tumor number or tumor size.

\section{Higher GSTA1 indicated better OS and DFS}

In $90 \mathrm{HCC}$ cases with prognostic information, we observed that GSTA1 was positively associated with OS (Figure 2A Left). Patients with higher GSTA1 had longer OS time, while low GSTA1 groups showed shorter OS. GSTA1 was also positively associated with DFS (Figure 2A Right). Patients with higher GSTA1 had longer DFS (median DFS $=64.27$ months for high GSTA1 group and 55.37 months for moderate GSTA1 group), while lower GSTA1 patients had shorter DFS (median DFS $=23.73$ months; $P<0.05$ ).

The prognostic value of GSTA1 was further confirmed by stratified OS and DFS analyses. Higher GSTA1 was correlated with longer OS (Figure 2B Left) and DFS (Figure 2B Right) in single tumor number and TNM stage I+II (all $P<0.05$ ). The pathological grade I+II subgroup showed the same trend, but without statistical significance.

\section{GSTA1 maybe an independent prognostic factor for HCC}

Univariate analysis showed that GSTA1, AFP, tumor number, tumor size, PVTT, and TNM stage were related to OS (Table 2) and DFS (Table 3) in HCC patients. Multivariate analysis was performed using the Cox Proportional hazards model and the analysis revealed that GSTA1, AFP, tumor number, PVTT and TNM were independent prognostic factors for HCC (all $P<0.05$ ). 
A.

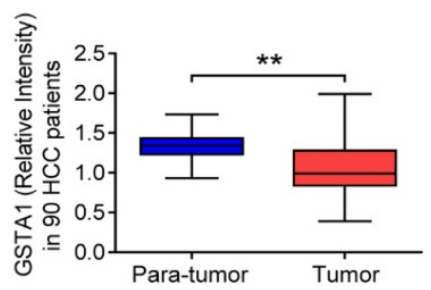

C.

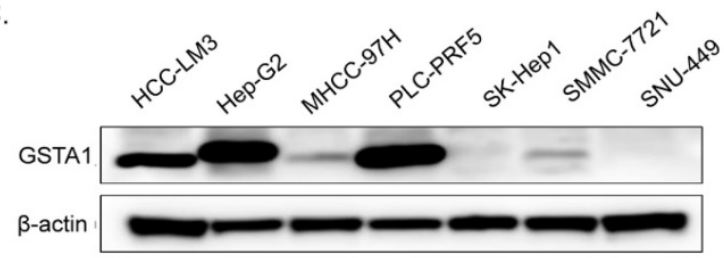

B. HCC patient (well differentiated)

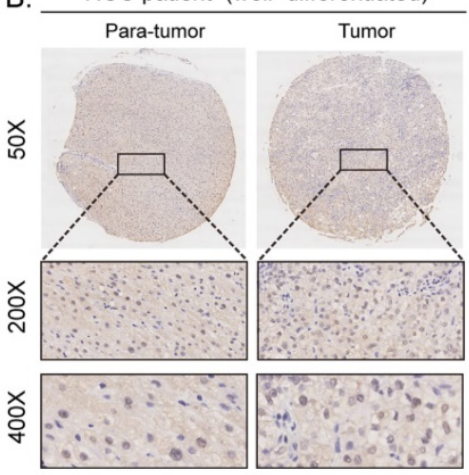

HCC patient (moderately differentiated)
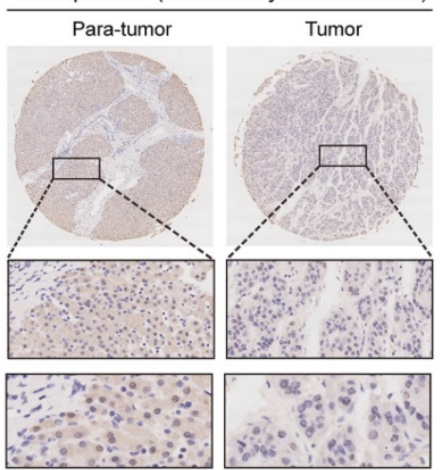

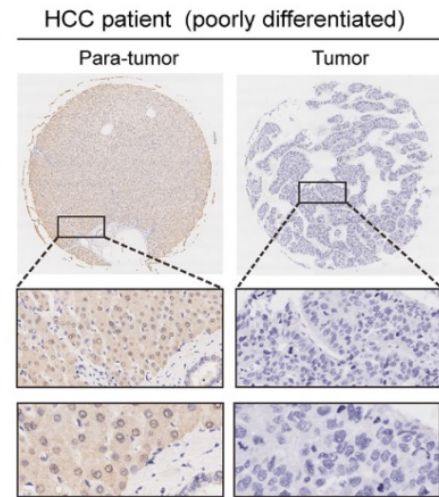

Figure 1: GSTA1 decreased in HCC. A. GSTA1 is downregulated in HCC tumor tissues compared with para-tumor tissues, calculated by Image Pro Plus (IPP) Image Analysis Software (**P < 0.01). B. the protein level of GSTAl is related to pathological differentiation of HCC. GSTAl was highly expressed in well-differentiated HCC tumors, but decreased heavily and was almost absent in poorly differentiated tumor tissues. Representative photomicrographs showed immunostaining of GSTA1 in well (Left), moderately (Middle) and poorly (Right) differentiated HCC specimens (magnification, 50x, 200x, 400x). C. Deficiency of GSTA1 was detected in liver cancer cell lines, including HCC-LM3 MHCC-97H, SK-Hep1, SMMC-7721 and SNU-449, but not in HepG2 and PLC-PRF5, checked by western blot.

Table 1. Correlation between GSTAl and clinicopathologic features in $90 \mathrm{HCC}$ patients

\begin{tabular}{|c|c|c|c|c|c|}
\hline \multirow[t]{2}{*}{ Variable (missing cases) } & \multirow[t]{2}{*}{ Cases } & \multicolumn{3}{|l|}{ GSTA1 } & \multirow[t]{2}{*}{$P$-value } \\
\hline & & Low & Moderate & High & \\
\hline Gender & & & & & 0.524 \\
\hline Female & $20(22.2 \%)$ & $7(35.0 \%)$ & $7(35.0 \%)$ & $6(30.0 \%)$ & \\
\hline Male & $70(77.8 \%)$ & $14(20.0 \%)$ & $37(52.8 \%)$ & $19(27.2 \%)$ & \\
\hline Age/year & & & & & 0.271 \\
\hline$\leq 50$ & $40(44.4 \%)$ & $12(30.0 \%)$ & $18(45.0 \%)$ & $10(25.0 \%)$ & \\
\hline$>50$ & $50(55.6 \%)$ & $9(18.0 \%)$ & $26(52.0 \%)$ & $15(30.0 \%)$ & \\
\hline HBsAg & & & & & 0.928 \\
\hline Negative & $19(21.1 \%)$ & $8(42.1 \%)$ & $9(47.4 \%)$ & $2(10.5 \%)$ & \\
\hline Positive & $71(78.9 \%)$ & $13(11.2 \%)$ & $35(26.4 \%)$ & $23(43.1 \%)$ & \\
\hline Preoperative AFP & & & & & $0.026^{*}$ \\
\hline$\leq 400 \mathrm{ng} / \mathrm{mL}$ & $56(62.2 \%)$ & $7(12.5 \%)$ & $32(57.1 \%)$ & $17(30.4 \%)$ & \\
\hline$>400 \mathrm{ng} / \mathrm{mL}$ & $34(37.8 \%)$ & $14(41.2 \%)$ & $12(35.3 \%)$ & $8(23.5 \%)$ & \\
\hline Tumor Number & & & & & 0.055 \\
\hline Single & $77(86.6 \%)$ & $16(20.8 \%)$ & $37(48.0 \%)$ & $24(31.2 \%)$ & \\
\hline Multiple & $13(13.4 \%)$ & $5(38.5 \%)$ & $7(53.8 \%)$ & $1(7.7 \%)$ & \\
\hline Tumor size & & & & & 0.307 \\
\hline$\leq 3 \mathrm{~cm}$ & $32(35.6 \%)$ & $5(15.6 \%)$ & $17(53.1 \%)$ & $10(31.3 \%)$ & \\
\hline $3-5 \mathrm{~cm}$ & $22(24.4 \%)$ & $6(27.3 \%)$ & $10(45.5 \%)$ & $6(27.2 \%)$ & \\
\hline$>5 \mathrm{~cm}$ & $36(30.0 \%)$ & $10(27.8 \%)$ & $17(47.2 \%)$ & $19(25.0 \%)$ & \\
\hline PVTT & & & & & $0.005^{\star * *}$ \\
\hline Absent & $58(64.4 \%)$ & $9(15.5 \%)$ & $31(53.4 \%)$ & $18(31.1 \%)$ & \\
\hline Present & $32(35.6 \%)$ & $12(37.5 \%)$ & $13(40.6 \%)$ & $7(21.9 \%)$ & \\
\hline Differentiation & & & & & $0.026^{*}$ \\
\hline Well & $9(10.0 \%)$ & $3(33.3 \%)$ & $1(11.1 \%)$ & $5(55.6 \%)$ & \\
\hline Moderate & $45(50.0 \%)$ & $5(11.1 \%)$ & $27(60.0 \%)$ & $13(28.9 \%)$ & \\
\hline Poor & $36(40.0 \%)$ & $13(36.1 \%)$ & $16(44.4 \%)$ & $7(19.5 \%)$ & \\
\hline TNM Stage & & & & & $0.011^{*}$ \\
\hline I & $56(62.2 \%)$ & $6(10.7 \%)$ & $32(57.1 \%)$ & $18(32.2 \%)$ & \\
\hline II & $20(22.2 \%)$ & $11(55.0 \%)$ & $5(25.0 \%)$ & $4(20.0 \%)$ & \\
\hline III-IV & $14(15.6 \%)$ & $4(28.6 \%)$ & $7(50.0 \%)$ & $3(21.4 \%)$ & \\
\hline
\end{tabular}

${ }^{*} P<0.05$, ** $P<0.01$.

Abbreviations: GSTA1: Glutathione S-transferase A1. HBsAg: hepatitis B surface antigen. AFP: alpha-fetoprotein. PVTT: portal vein tumor thrombosis. TNM: tumor-node-metastasis. 

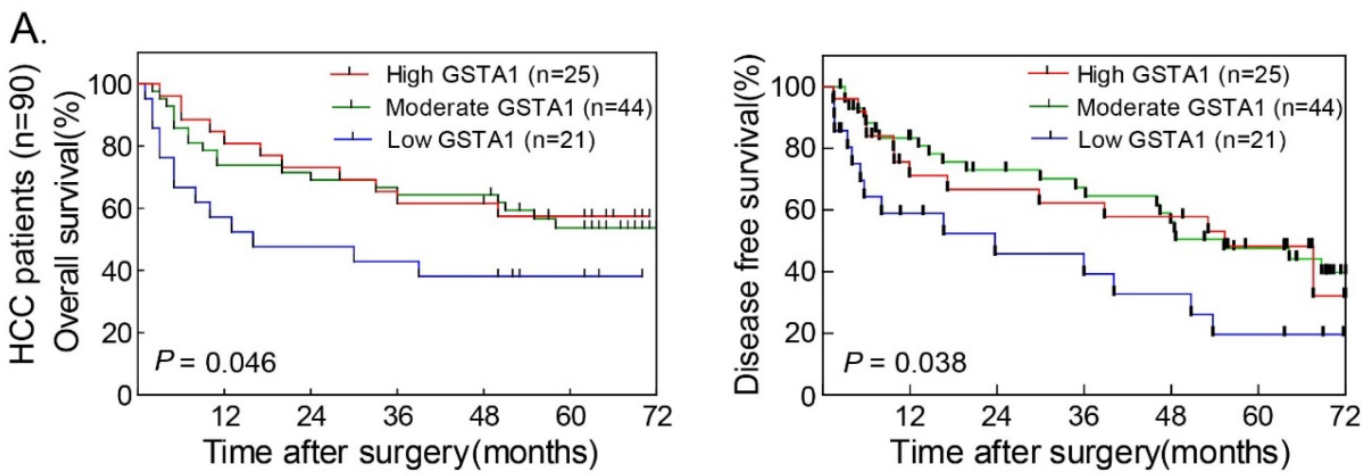

B.
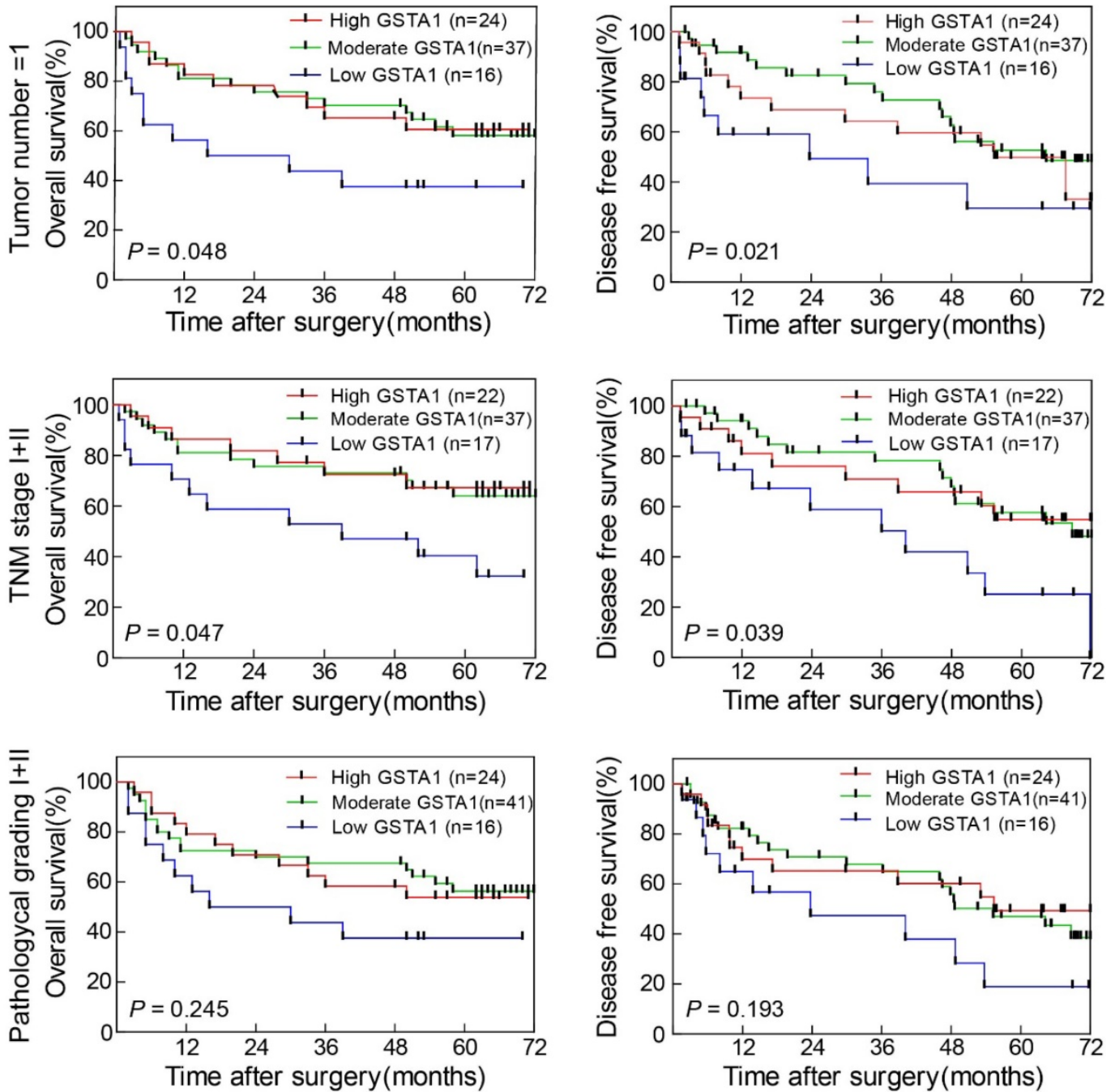

Figure 2: Higher GSTAI indicated better OS and DFS in HCC. A. Patients with higher GSTA1 had longer OS and DFS, while lower GSTAI indicated shorter OS and DFS $(* P<0.05)$. B. In single tumor number subgroup and TNM early stage subgroup, patients with high GSTA1 had long OS and DFS time (all $* P<$ $0.05)$. The pathological grade I+II subgroup showed the same trend, but without statistical significance $(P=0.245$ for OS and $P=0.193$ for $D F S$, respectively).

\section{GSTA1 overexpression inhibited hepatic cancer cell proliferation}

Liver cancer cells $97 \mathrm{H}$ and SNU449 (with a very low GSTA1) were chosen as experimental cells and were infected with the lentivirus vectors constitutively expressing GSTA1. CCK8 assays showed that OD450 of $97 \mathrm{H}$ in the control group was $0.363 \pm 0.052,0.666 \pm 0.079,1.179 \pm 0.104$ and $1.707 \pm$ 0.127 at $24,48,72$ and 96 hours, respectively. GSTA1 reduced the viability of $97 \mathrm{H}$ cells in a time-dependent manner, with OD450 of $0.303 \pm 0.050,0.547 \pm 0.047$, $0.784 \pm 0.281$, and $1.347 \pm 0.072$. A similar trend 
occurred in SNU449 cells (Figure 3A). Colony-formation assays were used to evaluate the long-term effect of GSTA1 on cell survival. GSTA1 overexpression led to a decrease in cell colony formation ability in $97 \mathrm{H}$ and SNU449 cells (all $P<$ 0.05 , Figure 3B).

Table 2. Univariate and multivariate analysis for predictors of OS in $90 \mathrm{HCC}$ patients

\begin{tabular}{|c|c|c|c|c|}
\hline \multirow[t]{3}{*}{ Variables } & \multicolumn{4}{|l|}{ OS } \\
\hline & \multicolumn{4}{|c|}{ Univariate Multivariate } \\
\hline & $P$-value & $\mathrm{HR}$ & $95 \% \mathrm{CI}$ & $P$-value \\
\hline Gender (Female vs Male) & 0.234 & - & - & - \\
\hline $\begin{array}{l}\text { Age/year ( } \leq 50 \text { ys vs }>50 \\
\text { ys) }\end{array}$ & 0.180 & - & - & - \\
\hline $\begin{array}{l}\text { HBsAg (Negative } v s \\
\text { Positive) }\end{array}$ & 0.932 & - & - & - \\
\hline $\begin{array}{l}\operatorname{AFP}(\mathrm{ng} / \mathrm{mL})(\leq 400 v s> \\
400)\end{array}$ & $0.000^{* *}$ & 2.641 & $1.387-5.025$ & $0.003^{* *}$ \\
\hline $\begin{array}{l}\text { Number of tumors (Single } \\
\text { vs Multiple) }\end{array}$ & $0.022^{*}$ & 2.174 & $1.036-4.560$ & $0.040^{*}$ \\
\hline $\begin{array}{l}\text { Tumor size } \mathrm{d} / \mathrm{cm}(\leq 5 \text { vs }> \\
\text { 5) }\end{array}$ & $0.005^{\star *}$ & 1.635 & $1.137-2.351$ & $0.008^{* *}$ \\
\hline $\begin{array}{l}\text { Pathological grade (I vs II vs } \\
\text { III-IV) }\end{array}$ & $0.015^{*}$ & 1.752 & $1.044-2.942$ & $0.034^{*}$ \\
\hline PVTT (Present vs Absent) & $0.000^{* *}$ & 2.805 & $1.503-5.236$ & $0.001^{* *}$ \\
\hline TNM (I vs II vs III-IV) & $0.000^{* *}$ & 2.566 & $1.721-3.826$ & $0.000^{* *}$ \\
\hline $\begin{array}{l}\text { GSTA1 (Low vs Moderate vs } \\
\text { High) }\end{array}$ & $0.036^{*}$ & 2.675 & $1.853-4.192$ & $0.047^{*}$ \\
\hline \multicolumn{5}{|l|}{${ }^{*} P<0.05,{ }^{* *} P<0.01$} \\
\hline \multicolumn{5}{|c|}{$\begin{array}{l}\text { Abbreviations: OS: overall survival. HR: hazard radio. CI: confidence interval. } \\
\text { HBsAg: hepatitis B surface antigen. AFP: alpha-fetoprotein. PVTT: portal vein } \\
\text { tumor thrombosis. TNM: tumor-node-metastasis. GSTA1: Glutathione } \\
\text { S-transferase a1. }\end{array}$} \\
\hline
\end{tabular}

Table 3. Univariate and multivariate analysis for predictors of DFS in $90 \mathrm{HCC}$ patients

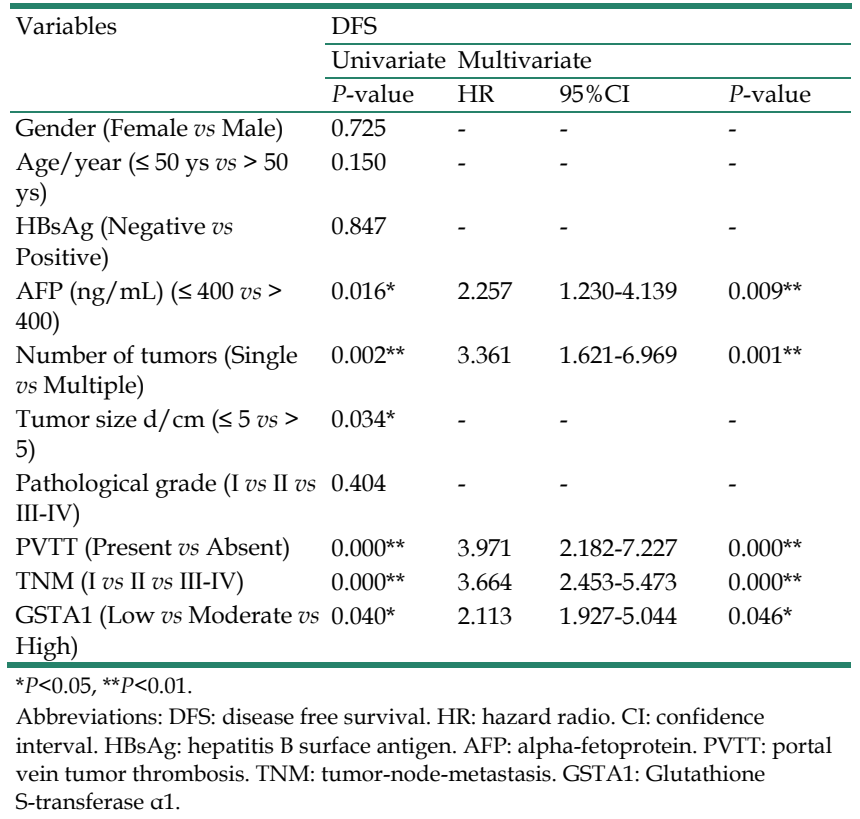

GSTA1 overexpression reduced hepatic cancer cell migration and invasion abilities

The migration and invasion abilities of liver cancer cells (97H and SNU449) were weakened in GSTA1 groups. Migration assays showed that the numbers of migratory cells were much less in GSTA1 groups than those in control groups. In invasion assays, the numbers of cells passed through the matrigel in GSTA1 groups were $26.00 \pm 8.54$ for $97 \mathrm{H}$ and $63.18 \pm 4.22$ for SNU449, and less than that in control groups $(38.30 \pm 10.05$ for $97 \mathrm{H}$ and $93.14 \pm 7.17$ for SNU449), all $P<0.05$, Figure 3C and 3D.

\section{GSTA1 may regulate the AMPK/mTOR pathway}

In GSTA1-overexpression groups, western blot showed an increased abundance of GSTA1 compared with the control groups, indicating that GSTA1 had been expressed stably and effectively in $97 \mathrm{H}$ and SNU449. GSTA1 overexpression upregulated LKB1 and p-AMPKa Thr172, without too much effect on total AMPK, but downregulated p-mTOR Ser2448, p-p70 S6K and MMP-9 (all $P<0.05$, Figure 3E).

\section{Discussion}

Previous GSTA1 studies have reached contradicting conclusions, so we investigated GSTA1's effects on tumor progression and prognosis in HCC. We began our study by exploring GSTA1 in tissue microarray using IHC. Analysis showed that GSTA1 abundances were lower in HCC tissues than that in adjacent para-tumor liver tissues. And patients with poorer differentiation had much lower GSTA1. These results align with those of Hayes $\mathrm{PC}^{18}$ and Campbell JA ${ }^{19}$, which found decreased GSTA1 activity in HCC tissue. GSTA1 is a kind of toxicide that can expel cytotoxic or reactive oxygen species (ROS) from the body. At the beginning of tumorigenesis, ROS accumulate slightly, and could be eliminated by enzymatic or nonenzymatic antioxidant easily ${ }^{20,21}$. However, with the progression of tumors, ROS increased, and antioxidants were suppressed in carcinoma $^{22}$. Therefore, we speculated that the decrease of GSTA1 in advanced hepatocellular carcinoma may be mainly related to ROS accumulation which proved to be able to accelerate the progression of $\mathrm{HCC}^{23}$.

We also observed that high GSTA1 was correlated with PVTT absence and low serum AFP. Both these factors occur during the early stage of tumorigenesis. And the number of PVTT or the serum AFP increased concomitantly with tumor progression in most HCC cases $^{24}$. Besides, we also found that GSTA1 was related to TNM stage in primary HCC. The earlier the stage, the higher the expression of 
GSTA1. The later the stage, the lower the expression of GSTA1. It was also very interesting that liver cancer cell lines with stronger metastasis ability had a lower GSTA1 abundance compared with other cells, which showed less metastatic potential. So, we suggested that GSTA1 may act as a biomarker in the progression of HCC, and the decrease of GSTA1 may indicate distant metastasis of the tumor and bad prognosis for HCC patients.
A.
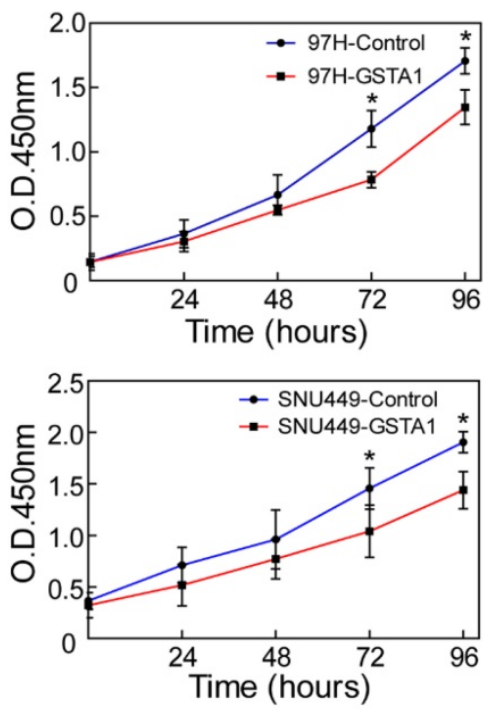

C.

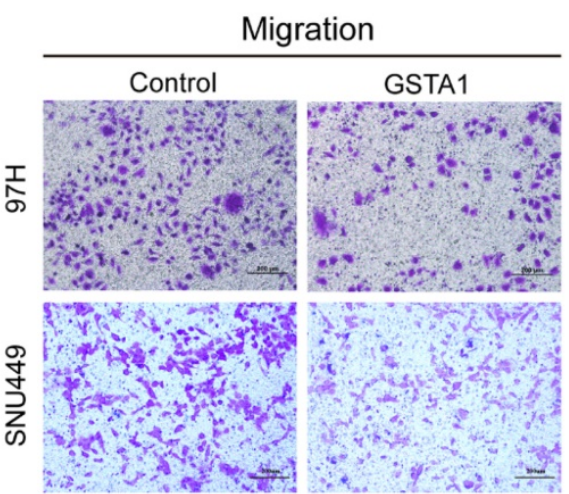

D.

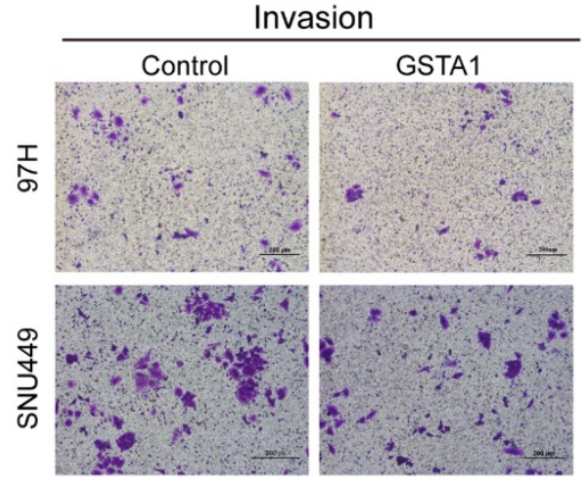

B.

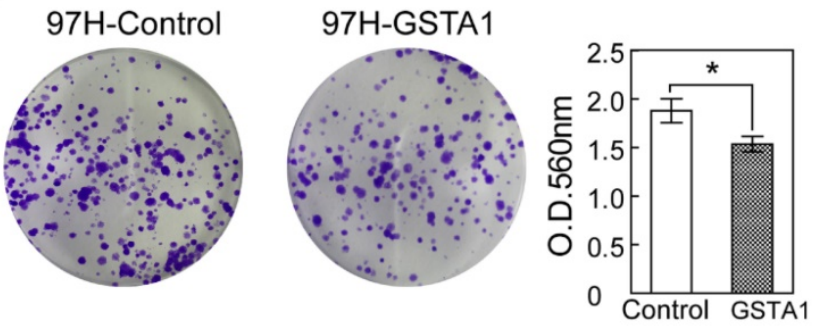

SNU449-Control SNU449-GSTA1
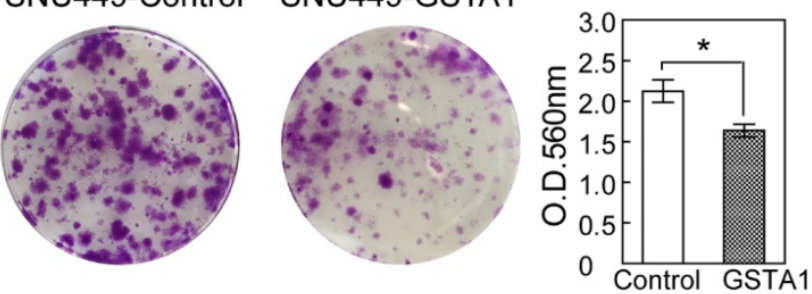

E.

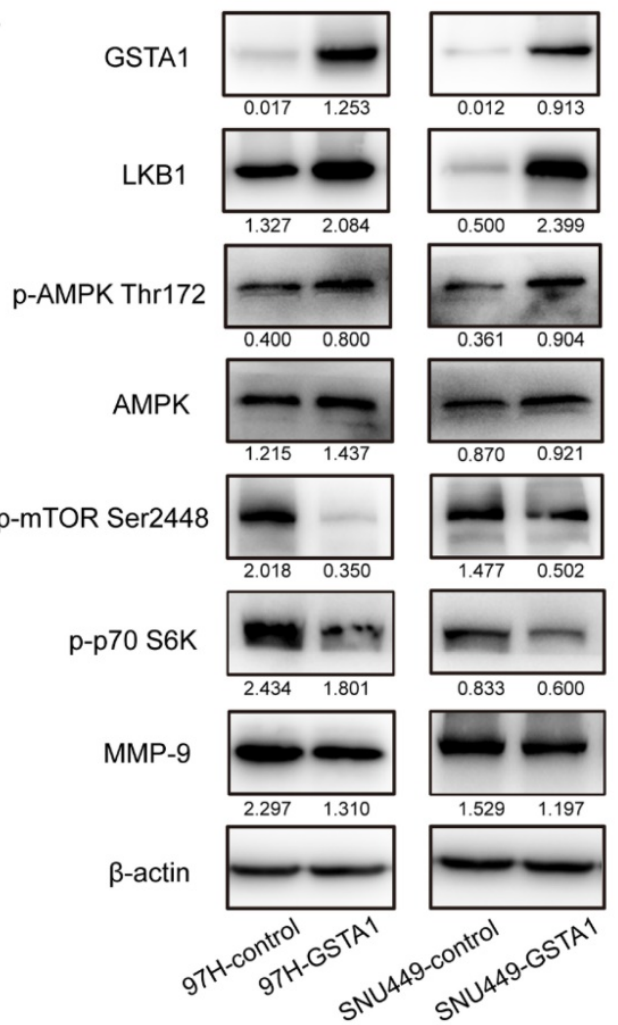

Figure 3: GSTA1 overexpression decreased liver cancer cell proliferation, migration and invasion abilities. A. CCK8 assay showed that GSTA1-transfected cells had decreased cell viability compared with empty vector control cells $(* P<0.05)$. B. Colony size and density in GSTA1 groups were smaller and rarer than those in control groups $(* P<0.05)$. The abilities of migration (C.) and invasion (D.) of liver cancer cells transfected with GSTA1 were decreased, which were detected by trans-well assays $(* P<0.05)$. Representative images were selected randomly from 5 fields (crystal violet staining, 400x) in each group and quantified by mean of five random fields. E. GSTA1 overexpression downregulated AMPK/mTOR. Western blot showed that GSTAl protein was overexpressed in GSTA1-transfected liver cancer cells, indicating a successful transfection. In 97H and SNU449 cells, GSTA1 overexpression increased LKB1 and p-AMPK Thr172 protein expression while the total AMPK amount remained unchanged. Western blotting results indicated that p-mTOR 2448 decreased in cells overexpressing GSTA1, compared with controls. Besides, p-p70 S6K as well as MMP-9 were downregulated in the GSTA1 groups. 
The clinical significance suggests that GSTA1 might influence the biological behavior of HCC. We found that GSTA1 suppresses hepatic cancer cell growth in both a short time and a long period, with a downregulation of p70 S6K (p70 ribosomal protein S6 kinases), which could promote elongation fator-1a (EF-1a) and Poly A-binding protein (PABP ${ }^{25}$. Besides, compared with control groups, GSTA1 overexpression cells showed much weak mobility in migration and invasion assays, and decreased abundance of MMP-9. Matrix metalloproteinases (MMPs) can degrade almost all the components of extracellular matrix (ECM) and destroy the histological barrier during the invasion, and play an important role in the metastasis of tumors 26,27 . MMP-9 is upregulated in HCC and is a marker of a bad prognosis ${ }^{28}$. So, these results suggest that downregulation of MMP-9 caused decreased metastasis ability of GSTA1 overexpressed cells.

Liver kinase B1(LKB1), an important upstream gene of adenylate-activated protein kinase (AMPK), acts as a tumor suppressor ${ }^{29}$. Previous experiments have shown that LKB1 can activate AMPK and then negatively regulate $\mathrm{mTOR}$, a key biological macromolecule that promotes cell metabolism and growth $^{30}$. In the process of tumorigenesis, decrease or absence of LKB1 or p-AMPK could activate p-mTOR, which phosphorylates S6K and 4EBP1, accelerates cell cycle, enhances cell proliferation, and ultimately accelerates tumorigenesis.

Our results showed that GSTA1 overexpression decreased HCC cell proliferation and significantly reduced their migration and invasion. It has been reported that glutathione deficiency is associated with LKB1 loss under oxidative stress ${ }^{29}$. We speculated that as a vital assistant of GSH, GSTA1 may cause LKB1 upregulation, which activated AMPK and reduced p-mTOR, thereby inhibiting cell proliferation and migration and invasion. In this experiment, increased LKB1 had a significant effect on AMPK/mTOR/MMP-9. Zhuang, et al. ${ }^{31}$ also found that high expression of LKB1 could reduce the expression of MMPs and inhibit the metastasis of breast cancer cells. So, it is reasonable that LKB1 inhibits the metastasis of GSTA1 overexpressed liver cancer cells by downregulating the expression of MMP-9.

In summary, better pathological differentiation of HCC indicated higher GSTA1 in tumors. And patients with higher GSTA1 were more likely to have better prognosis or stay in early stage HCC. We found that GSTA1 overexpression could inhibit liver cancer cell proliferation and metastasis through regulating LKB1/AMPK/mTOR directly or indirectly. All these results indicated GSTA1 could be applied as a potential prognostic biomarker and a new therapeutic target in HCC.

\section{Acknowledgements}

This study was supported by National Natural Science Foundation of China (No. 81902834, No. 81702857) as well as Hui-Chun Chin and Tsung-Dao Lee Chinese Undergraduate Research Endowment (CURE Program No. 18920, No. 19940), and Fudan's Undergraduate Research Opportunities Program (No. 18052). We would like to thank LetPub (www.letpub.com) for providing linguistic assistance during the preparation of this manuscript.

\section{Competing Interests}

The authors have declared that no competing interest exists.

\section{References}

1. Chen W, Zheng R, Baade PD et al. Cancer statistics in China, 2015. CA Cancer J Clin 2016;66:115-132.

2. Allocati N, Masulli M, Di Ilio C, Federici L. Glutathione transferases: substrates, inihibitors and pro-drugs in cancer and neurodegenerative diseases. ONCOGENESIS 2018;7:8.

3. Hayes JD, Flanagan JU, Uowsey IR. Glutathione transferases. Annu Rev Pharmacol Toxicol. 2005; 45:51-88

4. Mannervik B, Board PG, Hayes JD, et al. Nomenclature for mammalian soluble glutathione transferases. Methods Enzymol. 2005; 401:1-8.

5. Liu H, Yang Z, Zang L et al. Downregulation of Glutathione S-transferase A1 suppressed tumor growth and induced cell apoptosis in A549 cell line. ONCOL LETT 2018;16:467-474.

6. Pan XD, Yang ZP, Tang QL et al. Expression and function of GSTA1 in lung cancer cells. Asian Pac J Cancer Prev 2014;15:8631-8635.

7. Coric VM, Simic TP, Pekmezovic TD et al. Combined GSTM1-Null, GSTT1-Active, GSTA1 Low-Activity and GSTP1-Variant Genotype Is Associated with Increased Risk of Clear Cell Renal Cell Carcinoma. PLOS ONE 2016;11:e160570.

8. Wang W, Liu F, Wang C, Wang C, Tang Y, Jiang Z. Glutathione S-transferase A1 mediates nicotine-induced lung cancer cell metastasis by promoting epithelial-mesenchymal transition. EXP THER MED 2017;14:1783-1788.

9. Akhdar H, El SS, Musso O et al. The rs3957357C $>$ T SNP in GSTA1 Is Associated with a Higher Risk of Occurrence of Hepatocellular Carcinoma in European Individuals. PLOS ONE 2016;11:e167543.

10. Savic-Radojevic A, Djukic T, Simic T et al. GSTM1-null and GSTA1-low activity genotypes are associated with enhanced oxidative damage in bladder cancer. REDOX REP 2013;18:1-7.

11. Adnan H, Antenos M, Kirby GM. The effect of menadione on glutathione S-transferase A1 (GSTA1): c-Jun N-terminal kinase (JNK) complex dissociation in human colonic adenocarcinoma Caco-2 cells. TOXICOL LETT 2012;214:53-62.

12. Adnan H, Quach H, MacIntosh K, Antenos M, Kirby GM. Low levels of GSTA1 expression are required for Caco-2 cell proliferation. PLOS ONE 2012;7:e51739.

13. Pljesa I, Berisavac M, Simic T et al. Polymorphic expression of glutathione transferases A1, M1, P1 and T1 in epithelial ovarian cancer: a Serbian case-control study. I BUON 2017:22:72-79.

14. Yu Y, Li X, Liang C et al. The relationship between GSTA1, GSTM1, GSTP1, and GSTT1 genetic polymorphisms and bladder cancer susceptibility: A meta-analysis. Medicine (Baltimore) 2016;95:e4900.

15. Qu K, Liu SS, Wang ZX et al. Polymorphisms of glutathione S-transferase genes and survival of resected hepatocellular carcinoma patients. World J Gastroenterol 2015;21:4310-4322.

16. Tan N, Liu Q, Liu X et al. Low expression of B-cell-associated protein 31 in human primary hepatocellular carcinoma correlates with poor prognosis. HISTOPATHOLOGY 2016;68:221-229.

17. Liu X, Tan N, Liao H et al. High GSTP1 inhibits cell proliferation by reducing Akt phosphorylation and is associated with a better prognosis in hepatocellular carcinoma. Oncotarget 2018;9;8957-8971.

18. Hayes PC, May L, Hayes JD, Harrison DJ. Glutathione S-transferases in human liver cancer. GUT 1991;32:1546-1549.

19. Campbell JA, Corrigall AV, Guy A, Kirsch RE. Immunohistologic localization of alpha, mu, and pi class glutathione S-transferases in human tissues. CANCER-AM CANCER SOC 1991;67:1608-1613. 
20. Nunes SC, Serpa J. Glutathione in Ovarian Cancer: A Double-Edged Sword. INT J MOL SCI 2018;19.

21. Larasati YA, Yoneda-Kato N, Nakamae I, Yokoyama T, Meiyanto E, Kato JY. Curcumin targets multiple enzymes involved in the ROS metabolic pathway to suppress tumor cell growth. Sci Rep 2018;8:2039.

22. Sha Li, Hor-Yue Tan, Ning Wang, et al. The Role of Oxidative Stress and Antioxidants in Liver Diseases. Int J Mol Sci. 2015; 16: 26087-26124.

23. Eun HS, Cho SY, Joo JS et al. Gene expression of NOX family members and their clinical significance in hepatocellular carcinoma. Sci Rep 2017;7:11060.

24. Peng SY, Wang XA, Huang CY et al. Better surgical treatment method for hepatocellular carcinoma with portal vein tumor thrombus. World J Gastroenterol 2018;24:4527-4535.

25. Hay N, Sonenberg N. Upstream and downstream of mTOR. Genes Dev 2004;18:1926-1945.

26. Hernandez-Gea V, Toffanin S, Friedman SL, Llovet JM. Role of the microenvironment in the pathogenesis and treatment of hepatocellular carcinoma. GASTROENTEROLOGY 2013;144:512-527.

27. Affo S, Yu LX, Schwabe RF. The Role of Cancer-Associated Fibroblasts and Fibrosis in Liver Cancer. Annu Rev Pathol 2017;12:153-186.

28. Wang $\mathrm{Q}, \mathrm{Yu} \mathrm{W}$, Huang $\mathrm{T}$, Zhu $\mathrm{Y}$, Huang C. RUNX2 promotes hepatocellular carcinoma cell migration and invasion by upregulating MMP9 expression. ONCOL REP 2016;36:2777-2784.

29. Shaw RJ, Kosmatka M, Bardeesy N et al. The tumor suppressor LKB1 kinase directly activates AMP-activated kinase and regulates apoptosis in response to energy stress. Proc Natl Acad Sci U S A 2004;101:3329-3335.

30. Matter MS, Decaens T, Andersen JB, Thorgeirsson SS. Targeting the mTOR pathway in hepatocellular carcinoma: current state and future trends. I HEPATOL 2014;60:855-865.

31. Zhuang ZG, Di GH, Hou YF et al. Relationship between LKB1 gene and invasion-related factors of breast cancer cells. Zhonghua Yi Xue Za Zhi 2007;87:81-84 